\title{
COMPORTAMIENTO AGRESIVO EN VARONES DE 10 A 12 AÑOS, PERTENECIENTES A COLEGIOS DE VALPARAÍSO ${ }^{1}$
}

\section{AGGRESSIVE BEHAVIOR IN MALE 10 TO 12 YEARS OF BELONGING TO SCHOOLS VALPARAÍSO}

\author{
Liliana Pastén I. * \\ Pamela Lobos D. ${ }^{* *}$ \\ Angélica Mosqueda D. ${ }^{* *}$
}

\begin{abstract}
RESUMEN
Objetivos: Determinar la magnitud del comportamiento agresivo e identificar la distribución del nivel de agresividad según factores individuales, familiares e institucionales, en varones de 10-12 años de colegios municipalizados, particular-subvencionados y particular-pagados de Valparaíso. Material y Métodos: Estudio transversal, descriptivo-correlacional. Muestra correspondiente al 8,4\% del universo de matriculados en la comuna de Valparaíso, año 2006. Se aplicó una encuesta con preguntas cerradas, que midieron el comportamiento agresivo y los factores del estudio. Se establecieron cuatro niveles de agresividad: alto, mediano, bajo y sin agresividad. Resultados: $21,4 \%$ de los varones responde con un nivel alto o mediano de agresividad, frente a diversas situaciones. Respecto a los factores analizados, aquellos estadísticamente significativos a nivel individual fueron consumo de alcohol y drogas, que se presenta entre varones con más altos niveles de agresividad; a nivel familiar, el mayor porcentaje de alumnos que presentan alta agresividad han recibido castigo físico $(79,2 \%)$ y el patrón de crianza ejercido en sus familias es inconsistente (33,3\%); a nivel institucional, alumnos que tienen niveles altos de agresividad asisten en su mayoría a escuelas municipales $(83,3 \%)$ y pequeñas $(54,2 \%)$. Conclusión: conocer la magnitud del comportamiento agresivo y los factores asociados es fundamental para direccionar acciones de promoción y prevención de este comportamiento, especialmente por la/el enfermera/o, dado el contacto temprano y sistemático que sostiene con las familias en el Control de Salud Infantil, al observar el estilo de apego, indagar prácticas de crianza y fomentar patrones de crianza que permitan el logro de las tareas del desarrollo.
\end{abstract}

Palabras clave: Comportamiento infantil, violencia, agresión, instituciones académicas.

\begin{abstract}
Objective: To determine the extent of aggressive behavior and identify the distribution of level of aggressiveness as individual, family and institutional, in boys 10-12 years old from municipal schools, subsidized private and paid particular ones in Valparaiso District. Material and Method: Cross-sectional, descriptive correlational study. Sample corresponding to $8.4 \%$ of the universe of students enrolled in the district of Valparaíso, 2006. An inquiry with closed questions was used, divided into four parts measuring aggressive behavior and the factors studied. Four levels of aggressiveness were determined: high, medium, low and without aggressiveness. Results show that $21.4 \%$ of those children react to different situations with a high or medium level of aggressiveness. The factors statistically significant at an individual level were the use of alcohol and drugs, situation which is common among boys with a high level of aggressiveness. At a family level, the highest percentage of boys pre-

\footnotetext{
${ }^{1}$ Institución patrocinante: Dirección de Investigación y Proyectos Universidad de Valparaíso (DIPUV).

* Enfermera - Matrona, Magíster en Educación, Profesora Adjunto Universidad de Valparaíso, Valparaíso - Chile, e-mail: liliana.pasten@uv.cl

${ }^{* *}$ Enfermera - Matrona, Magíster en Enfermería, Profesora Auxiliar Universidad de Valparaíso, Valparaíso - Chile, e-mail: pamela.lobos@uv.cl

${ }_{* * *}$ Enfermera - Matrona, Especialista en investigación en el fenómeno de las drogas, Profesora Auxiliar Universidad de Valparaíso, Valparaíso - Chile, e-mail: angelica.mosqueda@uv.cl
} 
senting high aggressiveness has received physical punishment (79.2\%) and their upbringing pattern has been inconsistent $(33.3 \%)$. At an institutional level, the children with a high level of aggressiveness go mainly to municipal schools (83.3\%) and small schools (54.2\%). It is concluded that knowing the level of aggressive behavior and the associated factors is essential for directing the prevention of this behavior, especially by means of the nurses action who are the professionals keeping an early and systematic contact with the family at the Child Health Control, where they are able to observe the attachment style, to find out about upbringing practices and to stimulate upbringing patterns focused on the child's development.

Key words: Child behavior, violence, aggression, schools.

Fecha recepción: 21.11.09 Fecha aceptación: 09.04.11

\section{INTRODUCCIÓN}

El comportamiento agresivo se ha definido como una "Acción negativa desarrollada por alguien, quien de forma intencionada, causa daño, hiere o incomoda a otra persona" (1). Fernández y Serna (2) señalan que la agresividad y la violencia surgen del desequilibrio de la vida moderna; así también, la teoría sociológica la sitúa como parte del aprendizaje en un determinado contexto. La familia y la escuela por sus estructuras, formas de organización, jerarquizaciones y patrones de interacción, son los principales contextos modeladores del comportamiento de sus integrantes, por lo tanto, es aquí donde se pueden producir alteraciones en la socialización, generando como resultado un comportamiento desadaptativo, principalmente entre sus miembros en formación.

En este sentido, la violencia se ha convertido en una forma de relación comunicativa funcional en las escuelas (3), en la que se observan roles, jerarquías de poder, espacios y tiempos de convivencia. Es considerada un proceso interpersonal asimétrico que afecta al menos a dos protagonistas: a quien la ejerce $\mathrm{y}$ a quien la padece, aunque se ha distinguido un tercer componente: quien la contempla sin poder o querer evitarla (4). La agresión física o psíquica, ejercida de forma persistente en el tiempo, por uno o más escolares sobre otro que no sabe cómo defenderse, ha sido definida internacionalmente con el término inglés bullying; en la literatura hispana especializada se realiza una diferenciación de términos, denominando "intimidación" a la violencia psicológica y "matonaje" a la agresión física.

Diversos acontecimientos impactantes sobre violencia en las escuelas, difundidos a través de los medios de comunicación, han contribuido a instalar en el imaginario social la idea de que este fenómeno ha ido en aumento. Su estudio surge en los países escandinavos a inicios de los años 70, siendo el psicólogo noruego Olweus (1) el pionero en la investigación de esta materia. En la década de los 90 se extiende a otros países europeos y los Estados Unidos (5); en América Latina, Brasil aparece como el primer país en preocuparse por esta problemática con un estudio realizado por Abramovay y Rua publicado el año 2002 (6); en Chile, diversos autores citan la investigación realizada por Contador en el año 2001 como la primera experiencia cuantitativa, que explora directamente el tema de la violencia escolar en el país $(7,8)$.

Los estudios de prevalencia realizados investigan la percepción de los estudiantes respecto a su participación como agresor, agredido o como observador en hechos de violencia: Olweus (1) encontró que un 15\% de los estudiantes nórdicos decía haber participado en alguna conducta violenta hacia un compañero; Smokowski y Kopasz (9) mostró que uno de cada tres estudiantes han sufrido bullying de parte de sus compañeros en los Estados Unidos; Abramovay (10) en Brasil encontró que el 30\% de los estudiantes de colegios públicos han recibido agresión 
física; en Chile, la encuesta de violencia en el ámbito escolar realizada por el Ministerio del Interior en el año 2007 (11) muestra que el 26,3\% de los encuestados reconoce haber sido agredido en el último año, en comparación con un 45,2\% encontrado en el año 2005.

Asimismo, se han desarrollado investigaciones tendientes a indagar respecto al impacto en la salud física y mental de los escolares expuestos a altos niveles de violencia, identificando sintomatología común entre los niños que han sido víctimas de bullying: bajo bienestar psicológico, pobre ajuste social, altos niveles de estrés y somatización de problemas psicológicos $(1,12-15)$.

Por lo tanto, si bien este problema surge en el ámbito educacional, también debe considerarse desde una perspectiva de salud, siendo posible analizarlo bajo el "modelo del sistema conductual" planteado por la enfermera norteamericana Dorothy Johnson (16), quien señala que cada persona es un sistema conductual vinculado con su entorno y cuando este sistema está en equilibrio genera una conducta funcional.

El abordaje de esta problemática debe ser multidisciplinario, en ella la enfermera/o ocupa una posición estratégica por su continuo contacto a través de los programas de Salud Infantil y Escolar, donde, como señala Johnson: "puede utilizar la protección, el cuidado y la estimulación para mantener el equilibrio de las personas y ayudar a generar un nivel de funcionamiento óptimo" (16).

Expertos en el tema postulan que la prevención de las conductas antisociales pueden optimizarse a través de la promoción temprana de la conducta prosocial (17), por lo tanto, realizar una intervención a través de guías anticipatorias dirigida a los padres o cuidadores, profesores o directamente a los niños y adolescentes, debe ser una prioridad para el profesional enfermera/o.

El objetivo general del presente estudio es determinar la magnitud del comportamiento agresivo de los varones de 10 a 12 años pertenecientes a colegios de Valparaíso, con la finalidad de focalizar intervenciones en grupos de riesgo como parte del rol preventivo de la enfermera(o) en la salud infantil.

La elección de varones como sujeto de estudio se fundamenta en múltiples investigaciones, nacionales y extranjeras, que han mostrado que éstos presentan comportamientos más agresivos que las mujeres ( 1 , 5, 17-21). Asimismo, dado que en Chile se han realizado estudios mayoritariamente en enseñanza media, se ha decidido estudiar el fenómeno entre los 10 y 12 años.

Los objetivos específicos planteados son: identificar la distribución del nivel de agresividad del grupo en estudio e identificar los factores individuales, familiares e institucionales asociados en los varones con comportamiento agresivo.

En relación a las variables, se estudiaron las siguientes:

1. Nivel de agresividad: Para obtener los datos se utilizó un cuestionario de autorreporte de eventos de agresividad como respuesta del adolescente frente a diferentes situaciones.

\section{Factores individuales:}

2.1. Edad: Es un factor que se ha estudiado por su probable relación con el comportamiento agresivo desde dos puntos de vista: vivencia y percepción de intimidación. En este sentido, las investigaciones han mostrado que a mayor edad, tanto la cantidad de eventos agresivos como la percepción de violencia entre los estudiantes van disminuyendo $(5,18,22,23)$.

2.2. Rendimiento académico: La evidencia muestra que los estudiantes más agresivos tienen mayor tendencia a repetir de curso (20); por otro lado, en las escuelas en que el nivel académico es bajo se da mayor violencia entre los alumnos (21).

2.3. Posición ordinal: Se ha encontrado que 
los niños que ocupan un lugar intermedio entre los hermanos presentan actitudes menos agresivas que los niños mayores o menores (24).

2.4. Hábitos televisivos: Especialistas señalan que la mayor parte de la programación televisiva considera la violencia como una vía adecuada para conseguir determinados objetivos, lo cual es imitado por la población infantil (25). Se ha estudiado que los niños que más consumen películas de terror y acción son más agresivos (20). Otro estudio demostró que al reducir el tiempo de exposición de los niños a la televisión y los videojuegos, disminuye objetivamente la agresividad de sus conductas (26).

2.5. Consumo de alcohol y drogas: Se ha visto que alrededor de un tercio de los estudiantes que consumen drogas han participado o iniciado agresiones contra alguno de sus compañeros (27). Asimismo, se ha relacionado el consumo de alcohol con el comportamiento agresivo (15).

\section{Factores familiares:}

3.1. Tipo de familia: Respecto a las características de la familia a la que pertenecen los niños con comportamiento agresivo, en un estudio que abarca este tema (20) no se encontró diferencias entre familias biparentales y familias monoparentales.

3.2. Edad y escolaridad de los padres: No se han encontrado estudios que aporten información al respecto; desde el punto de vista de enfermería, nos parece necesario incorporarlas para conocer si éstas constituyen un factor de focalización de futuras intervenciones.

3.3. Patrón de crianza: Diversos investigadores, nacionales e internacionales, han encontrado que los niños y adolescentes que presentan conductas más agresivas han sido maltratados física o verbalmente por sus padres (14, 20, 28-30). Otros autores informan el uso de patrones de crianza que combinan la permisividad con el empleo de métodos autoritarios y coercitivos, en las familias de los niños considerados como agresivos (31).

\section{Factores institucionales:}

4.1. Dependencia administrativa: La información encontrada al respecto es diversa. Un estudio en Brasil muestra que las agresiones serían más frecuentes en colegios municipalizados (6); en cambio en Chile, la última encuesta de violencia escolar muestra que sería mayor en los colegios particulares pagados (11).

4.2. Tipo de establecimiento: Al respecto, es conocido que los varones tienen comportamientos más agresivos que las mujeres, por lo que se desea conocer si es posible extrapolar a colegios sólo de varones y si existen diferencias con los colegios mixtos.

4.3. Tamaño del colegio: La evidencia señala que las escuelas grandes reportan mayores índices de agresividad (20).

\section{MATERIAL Y MÉTODO}

Es una investigación cuantitativa, transversal, descriptivo-correlacional, realizada en 14 colegios municipalizados, particulares subvencionados y particulares pagados de Valparaíso.

El universo está constituido por 6.539 varones de 10 a 12 años matriculados al mes de julio del año 2006. La muestra representa un $8,4 \%$ del universo, conformada por 551 varones, seleccionados a través de un muestreo aleatorio simple.

El proyecto de investigación fue sometido a evaluación por parte del Comité de Ética de la Facultad de Medicina de la Universidad 
de Valparaíso, siendo aprobado en dicha instancia.

Los datos fueron obtenidos por medio de un cuestionario adaptado de un trabajo de investigación de pregrado ${ }^{2}$, el que se modificó de acuerdo a sugerencias de expertos; éste se divide en cuatro partes: 28 ítemes responden a las variables individuales, 17 a los factores familiares, 4 a los institucionales y 20 caracterizan el comportamiento de los adolescentes; previo a la aplicación se realizó una prueba piloto en 20 niños. La encuesta final fue aplicada a grupos de varones, supervisada por encuestadores capacitados, con previa solicitud del consentimiento informado, tanto a los padres como a los alumnos participantes.

Para determinar la confiabilidad del cuestionario se calculó el coeficiente alpha de Cronbach, cuyo resultado fue de 0,732 . La validez de contenido se estableció mediante juicio de expertos.

Para el análisis, los datos que miden el comportamiento agresivo se agrupan en cuatro categorías: alta, mediana, baja y no agresivo. Para construir la escala, a cada respuesta se le otorgó un puntaje de 0 a 5 , siendo 0 la alternativa que no expresa agresividad y 5 la de mayor grado de agresividad; según el número de preguntas con respuestas con mayor puntaje, se definió como alta agresividad cuando el adolescente respondió 8 o más preguntas con puntaje máximo; mediana 7 a 5 respuestas; baja de 4 a 3 respuestas y no agresivo entre 2 y 0.

Para la organización, el procesamiento y análisis de los datos se realizó con el software Microsoft Office Excel 2007 y Epidat 3.1 respectivamente, lo que permitió un análisis descriptivo de la información con tablas de frecuencia y de contingencia. Para medir relaciones entre las variables analizadas se utilizó la prueba de hipótesis de independencia
Chi-cuadrado de Pearson, utilizando una probabilidad de error de tipo 1 del 5\%.

\section{RESULTADOS}

La información contenida en la Tabla 1 destaca que el 21,4\% de los varones responde con un nivel alto o mediano de agresividad, frente a diversas situaciones.

A continuación se presenta la distribución porcentual de los varones, según factores individuales, familiares e institucionales, respecto de cada nivel de agresividad.

- Edad: Los varones de 10 años presentan los mayores porcentajes de alta y mediana agresividad, por otro lado, los de 11 años presentan el porcentaje más alto de baja agresividad y no agresivo. Sin embargo, el análisis estadístico no muestra significancia.

- Rendimiento académico: En general, se observa un buen rendimiento en los integrantes de la muestra; sin embargo, la tendencia muestra que el grupo de niños con alta agresividad tiene el porcentaje más alto de notas entre 4,0 y $4,9(18,8 \%)$ y el porcentaje más bajo de notas entre 6,0 y 7,0 (39,6\%), no obstante el estudio no encontró significancia estadística.

- Posición ordinal: Un tercio de los varones con alta agresividad $(31,3 \%)$ es el primero entre los hermanos, en cambio, un tercio de los niños con baja agresividad (32\%) y no agresivos $(32,5 \%)$ ocupan el segundo lugar entre sus hermanos. No se encontró significancia estadística.

- Hábitos televisivos: El número de horas

\footnotetext{
${ }^{2}$ Aguilera V, Cabrera K, Carrión M, Díaz P, Morales L, Marín M, Peña S, Urra D. “Manejo de las conductas agresivas de preescolares entre 3 y 5 años en la población de Valparaíso Consultorio Plaza Justicia. Seminario Tesis Carrera de Enfermería y Obstetricia.1999. Universidad de Valparaíso.
} 
que los adolescentes destinan a ver televisión presentó una distribución uniforme en los distintos niveles de agresividad, lo que no mostró significancia estadística. También se preguntó con quién ve televisión y quién decide el programa, en ambos casos no existe significancia estadística al relacionarlo con el nivel de agresividad de los adolescentes, sin embargo, destaca que en general la mitad de los niños $(51,2 \%)$ ve televisión solo y en su mayoría $(70,8 \%)$ son ellos los que deciden el programa que ven.

- Consumo de alcohol y drogas: Llama la atención que un 24,5\% (135 niños) ha consumido alcohol y un 2,0\% (11 niños) contesta que ha consumido drogas. Se observa que el mayor porcentaje de consumo de alcohol se da entre los varones con más altos niveles de agresividad y el mayor porcentaje de no agresivos corresponde a no consumidores de alcohol, lo que tiene una significancia estadística.

Con respecto al consumo de drogas es posible observar la misma tendencia que en el consumo de alcohol, es decir, los niños con mayor agresividad son los que tienen el más alto porcentaje de consumo de drogas y los niños no agresivos tienen el consumo más bajo, lo que también es estadísticamente significativo.

- Tipo de familia: Se observa una distribución proporcional entre familias nucleares $(60 \%)$ y extendida (40\%) entre los diferentes grupos de los varones del estudio, sin significancia estadística al relacionarlo con el nivel de agresividad.

$\mathrm{Al}$ analizar la existencia de uno o ambos padres en las familias de los varones, se encontró un leve aumento de familias monoparentales en los niños con alta agresividad $(37,5 \%)$ al compararlo con los otros grupos, tendencia que no mostró significancia estadística.
- Patrón de crianza: Respecto a esta variable, se analizó desde dos perspectivas: aplicación de castigo físico como medida disciplinaria y tipo de patrón de crianza.

Entre los adolescentes con un nivel de agresividad alto, el mayor porcentaje pertenece a familias cuyo patrón de crianza es inconsistente $(33,3 \%)$, en cambio, los niños que presentan baja agresividad o que no son agresivos, el mayor porcentaje pertenece a familias cuyo patrón de crianza predominante es el democrático ( $47 \%$ y $46,4 \%$ respectivamente), esta asociación indicó significancia.

En general se observa que un 48,8\% de los varones de la muestra han recibido castigo físico por parte de algún adulto, de éstos, en el 94,8\% de los casos el castigo físico fue aplicado por alguno de los padres.

El mayor porcentaje de los niños que presentan alta agresividad han recibido castigo físico (79,2\%), en contraste con el mayor porcentaje de los niños no agresivos que no lo han recibido $(60,7 \%)$, esto presenta una relación significativa desde el punto de vista estadístico.

- Edad y escolaridad de los padres: En esta variable se incluyeron sólo los padres que viven con los adolescentes.

En relación a la edad de los padres, en todos los grupos se encontró que, tanto la madre como el padre, el mayor porcentaje se concentra en edades entre 30 y 50 años (82,6\% en ambos casos), no observándose significancia estadística al relacionarla con el nivel de agresividad de los niños.

Se estudió la escolaridad de la madre y el padre por separado. En relación con la escolaridad de la madre, el porcentaje más alto es de escolaridad media completa en todos los niveles de agresividad, lo que no muestra una significancia estadística.

Se destaca que el mayor porcentaje de escolares con niveles de agresividad alto, el padre tiene escolaridad media completa 
(56,7\%), lo que resultó estadísticamente significativo.

- Dependencia administrativa del colegio: Se aprecia que los alumnos que tienen niveles altos de agresividad pertenecen en su mayoría a escuelas municipales $(83,3 \%)$, lo que muestra asociación estadística.

- Tipo de establecimiento: Se observa que los estudiantes con un nivel alto de agresividad asisten a colegios mixtos. Esta tendencia no mostró significancia estadística.

- Tamaño del colegio: Es posible observar que el porcentaje más alto de niños con alta agresividad $(54,2 \%)$ estudian en colegios pequeños. Mientras que en los colegios grandes se concentran los porcentajes más altos de baja agresividad y no agresivos. La prueba de independencia indica que es significativo.

Tabla 1. Distribución de los varones del estudio en número y porcentaje, según nivel de agresividad.

\begin{tabular}{lcc}
\hline Nivel agresividad & Frecuencia & $\%$ \\
\hline NO AGRESIVO & 252 & 45,7 \\
\hline BAJO & 181 & 32,8 \\
\hline MEDIANO & 70 & 12,7 \\
\hline ALTO & 48 & 8,7 \\
\hline Total & 551 & 100 \\
\hline
\end{tabular}

Fuente: DIPUV Estudio sobre nivel de agresividad en varones de 10 a 12 años matriculados al mes de julio del año 2006 en colegios de Valparaíso, Chile.

Tabla 2. Distribución porcentual de los varones según nivel de agresividad en cada uno de los factores individuales y significancia estadística de su relación.

\begin{tabular}{|c|c|c|c|c|c|}
\hline \multirow{2}{*}{$\begin{array}{l}\text { FACTORES } \\
\text { INDIVIDUALES }\end{array}$} & \multicolumn{4}{|c|}{ Nivel agresividad (\%) } & \multirow[t]{3}{*}{ Valor $\mathbf{p}$} \\
\hline & ALTA & MEDIANA & BAJA & NO AGRESIVO & \\
\hline Total & 8,7 & 12,7 & 32,8 & 45,7 & \\
\hline \multicolumn{6}{|l|}{ Rango edad (años) } \\
\hline 10 & 39,6 & 38,6 & 27,6 & 36,9 & \multirow{4}{*}{$\mathrm{p}=0,46$} \\
\hline 11 & 37,5 & 35,7 & 44,2 & 38,5 & \\
\hline 12 & 22,9 & 25,7 & 28,2 & 24,6 & \\
\hline Total & 100 & 100 & 100 & 100 & \\
\hline \multicolumn{6}{|l|}{ Rendimiento académico } \\
\hline Menor a 4,0 & 0 & 0 & 1,1 & 0 & \multirow{6}{*}{$\mathrm{p}=0,24$} \\
\hline 4,0 a 4,9 & 18,8 & 8,6 & 8,3 & 6,7 & \\
\hline 5,0 a 5,9 & 41,7 & 44,3 & 41,4 & 43,3 & \\
\hline 6,0 a 7,0 & 39,6 & 45,7 & 49,2 & 49,6 & \\
\hline No contesta & 0 & 1,4 & 0 & 0,4 & \\
\hline Total & 100 & 100 & 100 & 100 & \\
\hline \multicolumn{6}{|l|}{ Posición ordinal en familia } \\
\hline Hijo único & 6,3 & 18,6 & 18,2 & 15,5 & \\
\hline Primer hijo & 31,3 & 22,9 & 21,5 & 24,2 & \\
\hline Segundo hijo & 27,1 & 25,7 & 32 & 32,5 & \\
\hline
\end{tabular}


Continuación Tabla 2.

\begin{tabular}{|c|c|c|c|c|c|}
\hline Tercer hijo & 16,7 & 20 & 12,7 & 16,7 & \multirow[t]{4}{*}{$\mathrm{p}=0,34$} \\
\hline Cuarto hijo o más & 16,7 & 12,9 & 15,5 & 9,9 & \\
\hline No contesta & 2,1 & 0 & 0 & 1,2 & \\
\hline Total & 100 & 100 & 100 & 100 & \\
\hline \multicolumn{6}{|l|}{ Horas viendo TV } \\
\hline 1 a 2 & 50 & 35,7 & 40,3 & 42,1 & \multirow{6}{*}{$\mathrm{p}=0,36$} \\
\hline Más de 2 a 4 & 16,7 & 17,1 & 29,8 & 31,3 & \\
\hline Más de 4 a 6 & 6,3 & 20 & 13,3 & 13,5 & \\
\hline Más de 6 & 27,1 & 25,7 & 16,6 & 12,7 & \\
\hline No contesta & 0 & 1,4 & 0 & 0,4 & \\
\hline Total & 100 & 100 & 100 & 100 & \\
\hline \multicolumn{6}{|l|}{ Consumo de alcohol } \\
\hline $\mathrm{Si}$ & 41,7 & 34,3 & 23,8 & 15,1 & \multirow{4}{*}{$\mathrm{p}=0,00$} \\
\hline No & 52,1 & 62,9 & 74,6 & 83,3 & \\
\hline No contesta & 6,3 & 2,9 & 0,6 & 2,4 & \\
\hline Total & 100 & 100 & 100 & 100 & \\
\hline \multicolumn{6}{|l|}{ Consumo de drogas } \\
\hline $\mathrm{Si}$ & 6,3 & 5,7 & 2,2 & 0 & \multirow{4}{*}{$\mathrm{p}=0,01$} \\
\hline No & 91,7 & 90 & 94,5 & 98,4 & \\
\hline No contesta & 2,1 & 4,3 & 3,3 & 1,6 & \\
\hline Total & 100 & 100 & 100 & 100 & \\
\hline
\end{tabular}

Fuente: Ídem Tabla 1.

Tabla 3. Distribución porcentual de los varones según nivel de agresividad en cada uno de los factores familiares y significancia estadística de su relación.

\begin{tabular}{|c|c|c|c|c|c|}
\hline \multirow[t]{2}{*}{ FACTORES FAMILIARES } & \multicolumn{4}{|c|}{ Nivel agresividad (\%) } & \multirow{3}{*}{ Valor $\mathbf{p}$} \\
\hline & ALTA & MEDIANA & BAJA & NO AGRESIVO & \\
\hline Total & 8,7 & 12,7 & 32,8 & 45,7 & \\
\hline \multicolumn{6}{|l|}{ Tipo de familia } \\
\hline Monoparental & 37,5 & 30,0 & 30,4 & 27,4 & \multirow{4}{*}{$\mathrm{p}=0,82$} \\
\hline Biparental & 62,5 & 68,6 & 68,5 & 70,6 & \\
\hline Otro tipo & 0,0 & 1,4 & 1,1 & 2,0 & \\
\hline Total & 100,0 & 100,0 & 100,0 & 100,0 & \\
\hline \multicolumn{6}{|l|}{ Patrón de crianza } \\
\hline Democrático & 27,1 & 38,6 & 47,0 & 46,4 & \multirow{6}{*}{$\mathrm{p}=0,02$} \\
\hline Autocrático & 20,8 & 15,7 & 14,4 & 19,4 & \\
\hline Permisivo & 12,5 & 15,7 & 6,1 & 2,8 & \\
\hline Castigador & 6,3 & 0,0 & 1,7 & 1,6 & \\
\hline Inconsistente & 33,3 & 30,0 & 30,9 & 29,8 & \\
\hline Total & 100,0 & 100,0 & 100,0 & 100,0 & \\
\hline \multicolumn{6}{|l|}{ Aplicación de castigo físico } \\
\hline No castigado & 20,8 & 48,6 & 47,0 & 60,7 & \multirow{3}{*}{$\mathrm{p}=0,0$} \\
\hline Castigador & 79,2 & 51,4 & 53,0 & 39,3 & \\
\hline Total & 100,0 & 100,0 & 100,0 & 100,0 & \\
\hline \multicolumn{6}{|l|}{$\begin{array}{l}\text { Rango de edad del Padre } \\
n=392 \text { con padre presente }\end{array}$} \\
\hline Menos de 30 & 16,7 & 8,0 & 10,2 & 8,6 & \multirow[b]{4}{*}{$\mathrm{p}=0,75$} \\
\hline 31 a 40 & 46,7 & 42,0 & 40,9 & 47,0 & \\
\hline 41 a 50 & 36,7 & 38,0 & 40,9 & 36,8 & \\
\hline Mayor de 50 & 0,0 & 12,0 & 6,3 & 6,5 & \\
\hline
\end{tabular}


Continuación Tabla 3.

\begin{tabular}{|c|c|c|c|c|c|}
\hline No contesta & 0,0 & 0,0 & 1,6 & 1,1 & \\
\hline Total & 100,0 & 100,0 & 100,0 & 100,0 & \\
\hline \multicolumn{6}{|l|}{ Escolaridad del Padre } \\
\hline Básica incompleta & 13,3 & 26,0 & 9,4 & 7,0 & \multirow{8}{*}{$\mathrm{p}=0,03$} \\
\hline Básica completa & 10,0 & 8,0 & 8,7 & 13,0 & \\
\hline Media incompleta & 10,0 & 6,0 & 16,5 & 11,9 & \\
\hline Media completa & 56,7 & 26,0 & 32,3 & 36,2 & \\
\hline Técnico superior & 3,3 & 10,0 & 11,8 & 8,6 & \\
\hline Profesional & 6,7 & 22,0 & 20,5 & 21,6 & \\
\hline No responde & 0,0 & 2,0 & 0,8 & 1,6 & \\
\hline Total & 100,0 & 100,0 & 100,0 & 100,0 & \\
\hline \multicolumn{6}{|l|}{$\begin{array}{l}\text { Rango de edad de la Madre } \\
n=507 \text { con madre presente }\end{array}$} \\
\hline Menos de 30 & 28,3 & 14,8 & 13,3 & 15,0 & \multirow{6}{*}{$\mathrm{p}=0,31$} \\
\hline 31 a 40 & 43,5 & 47,5 & 54,8 & 58,1 & \\
\hline 41 a 50 & 26,1 & 37,7 & 30,1 & 24,4 & \\
\hline Mayor de 50 & 2,2 & 0,0 & 1,2 & 1,3 & \\
\hline No contesta & 0,0 & 0,0 & 0,6 & 1,3 & \\
\hline Total & 100,0 & 100,0 & 100,0 & 100,0 & \\
\hline \multicolumn{6}{|l|}{ Escolaridad de la Madre } \\
\hline Básica incompleta & 6,4 & 16,4 & 10,2 & 7,3 & \multirow{8}{*}{$\mathrm{p}=0,47$} \\
\hline Básica completa & 13,0 & 9,8 & 7,8 & 9,4 & \\
\hline Media incompleta & 17,2 & 19,7 & 13,3 & 15,8 & \\
\hline Media completa & 45,5 & 21,3 & 40,4 & 38,0 & \\
\hline Técnico superior & 2,6 & 9,8 & 10,2 & 10,7 & \\
\hline Profesional & 13,0 & 21,3 & 16,3 & 17,1 & \\
\hline No responde & 2,6 & 1,6 & 1,8 & 1,7 & \\
\hline Total & 100,0 & 100,0 & 100,0 & 100,0 & \\
\hline
\end{tabular}

Fuente: Ídem Tabla 1.

Tabla 4. Distribución porcentual de los varones según nivel de agresividad en cada uno de los factores institucionales y significancia estadística de su relación.

\begin{tabular}{|c|c|c|c|c|c|}
\hline \multirow[t]{2}{*}{ FACTORES INSTITUCIONALES } & \multicolumn{4}{|c|}{ Nivel agresividad (\%) } & \multirow{3}{*}{ Valor $\mathbf{p}$} \\
\hline & ALTA & MEDIANA & BAJA & NO AGRESIVO & \\
\hline Total & 8,7 & 12,7 & 32,8 & 45,7 & \\
\hline \multicolumn{6}{|c|}{ Dependencia administrativa del Colegio } \\
\hline Municipal & 83,3 & 68,6 & 64,1 & 59,9 & \multirow{4}{*}{$\mathrm{p}=0,03$} \\
\hline Subvencionado & 16,7 & 31,4 & 32 & 34,9 & \\
\hline Particular & 0 & 0 & 3,9 & 5,2 & \\
\hline Total & 100 & 100 & 100 & 100 & \\
\hline \multicolumn{6}{|l|}{ Tipo de establecimiento } \\
\hline Mixto & 77,1 & 64,3 & 64,6 & 68,7 & \multirow{3}{*}{$\mathrm{p}=0,37$} \\
\hline Sólo hombres & 22,9 & 35,7 & 35,4 & 31,3 & \\
\hline Total & 100 & 100 & 100 & 100 & \\
\hline \multicolumn{6}{|l|}{ Tamaño del colegio } \\
\hline Pequeño (Menos de 400 alumno) & 54,2 & 34,3 & 29,3 & 29,4 & \multirow{4}{*}{$\mathrm{p}=0,04$} \\
\hline Mediano (401 a 800 a) & 20,8 & 27,1 & 28,7 & 32,1 & \\
\hline Grande $(801$ a 1.500 a) & 25 & 38,6 & 42 & 38,5 & \\
\hline Total & 100 & 100 & 100 & 100 & \\
\hline
\end{tabular}

Fuente: Ídem Tabla 1. 


\section{DISCUSIÓN Y COMENTARIO}

$\mathrm{Al}$ analizar los resultados obtenidos en el estudio, se encontró que más de la mitad de los niños $(53,2 \%)$ refirieron algún nivel de agresividad, entre los cuales el nivel alto corresponde a un $8,7 \%$ y el nivel medio a un $12,7 \%$. En estos últimos grupos habría que focalizar una intervención, por lo tanto nos parece importante conocer las características de él, tanto individuales, familiares e institucionales, las que presentaremos a continuación.

En primer lugar, respecto a las características individuales, destaca la tendencia en que los niños de menor edad presentan mayores porcentajes de alta y mediana agresividad, lo que concuerda con lo encontrado por otros investigadores, tanto nacionales como internacionales $(5,18,22,23)$.

Así también, sobre rendimiento académico, se observa que los niños más agresivos tienen el porcentaje más alto de notas entre 4,0 y 4,9 (12\% más que los niños no agresivos) y el porcentaje más bajo de notas entre 6,0 y 7,0 (10\% menos que los niños no agresivos) reflejando un rendimiento moderadamente menor, lo que concuerda con el estudio realizado por Funk en Alemania, donde determinó que los estudiantes más agresivos tenían rendimientos más bajos (con tendencia a repetir de curso) que aquellos que no presentaban este comportamiento (20).

Además, según la posición ordinal del varón entre sus hermanos, existe un predominio de hijos mayores entre los niños con nivel de agresividad alto (31,3\%), en cambio los niños con niveles más bajos de agresividad ocupan el segundo lugar entre ellos. Especialistas españoles plantean que los niños de posiciones intermedias son menos agresivos que los mayores o menores (24), por lo tanto podemos decir que los resultados tienen similitud, si bien la forma de abordar el estudio es distinta.

Aun cuando estudios en Alemania y los
Estados Unidos han informado de la directa relación existente entre el número de horas que los niños ven televisión y la agresividad $(20,26)$, en el presente estudio no se evidenció relación entre estos factores.

Debemos resaltar que la única variable individual que mostró significancia estadística es el consumo de alcohol y drogas, donde tanto los niños que refirieron consumir alcohol como los que indicaron haber consumido drogas se ubicaron en los niveles más elevados de agresividad. Lo anterior coincide con información entregada por el Consejo Nacional para el Control de Estupefacientes (CONACE), que ha encontrado que los estudiantes que usan drogas se involucran más en agresiones contra compañeros que los no consumidores (27). Respecto al alcohol, también hemos encontrado evidencia norteamericana que señala que a mayor consumo, existe un comportamiento más agresivo (15). Desde el punto de vista de Enfermería, la promoción de estilos de vida saludables y la prevención de consumo de alcohol y drogas es de gran relevancia, donde la/el enfermera/o que se desempeña en el área del niño y adolescente debe tener una capacitación en el tema, que le permita ser un agente promotor, realizando guías anticipatorias a los padres para que asuman un papel activo y enseñen a sus hijos a valorar la vida sana, a resistir la presión del grupo, a tomar decisiones responsables y a rechazar el alcohol y las drogas.

En segundo lugar, en cuanto a los aspectos familiares, no se observan diferencias en el nivel de agresividad de los varones según el tipo de familia a la que pertenecen, sea esta nuclear o extendida. Por otro lado, existe un leve aumento de niños con alta agresividad pertenecientes a familias monoparentales. Este resultado es distinto a lo comunicado en otro estudio, que informa no encontrar diferencias entre familias con uno o ambos padres (21).

Siguiendo con este aspecto, en relación a la edad y escolaridad de los padres, es imposible comparar los resultados encontrados 
con otras investigaciones, debido a que éste fue un elemento nuevo incorporado en este trabajo. Es importante señalar, como hallazgo de la investigación, que entre los varones con niveles de agresividad alta, el padre posee una escolaridad media completa $(56,7 \%)$, siendo estadísticamente significativo. Las autoras recomiendan profundizar este aspecto en una próxima investigación, con el propósito de indagar sobre los factores que podrían estar determinando esta situación.

En el mismo sentido, en el patrón de crianza, desde la perspectiva de aplicación de castigo físico, se observa que un alto porcentaje de varones con alta agresividad han recibido castigo, en contraste a los no agresivos que no lo han padecido, siendo estadísticamente significativo. Estos resultados corroboran lo encontrado en otros estudios, los cuales señalan que los niños con comportamiento agresivo han recibido maltrato, ya sea físico o verbal, por parte de sus padres $(14,20,28$ 30).

Asimismo, el análisis del tipo de patrón crianza muestra que cuando se aplica un patrón inconsistente, el adolescente tempranamente tiene un comportamiento más agresivo a diferencia de la ejecución de un patrón democrático, empleado en las familias de los varones menos agresivos, diferencia estadísticamente significativa. Este resultado es similar a otra investigación realizada por DíazAguado (31).

Este hallazgo es de gran interés para el profesional enfermera/o, quien está en contacto desde temprana edad con el/la niño/a y sus padres a través del Control de Salud, instancias en que puede observar el estilo y calidad de apego, indagar sobre las prácticas de crianza y fomentar un patrón de crianza basado en el afecto y disciplina, sin castigo físico ni psicológico, permitiendo la expresión de sentimientos en un ambiente cálido, de respeto mutuo, de aprendizaje positivo, que le permita al menor desarrollar tolerancia a las frustraciones, respetar la diversidad $y$ comunicarse en forma eficaz para incor- porarse en la sociedad de manera saludable. Los estudios $(18,20,32)$ indican que la falta de cariño y la poca comunicación con el niño/a pueden generar una persona violenta, debido a la falta de seguridad. Lo anterior es una preocupación de política pública, que es abordada por el Ministerio de Salud, a través del Programa Chile Crece Contigo (33).

Los padres se ven enfrentados a situaciones complejas durante el crecimiento y desarrollo de su hijo/a, necesitando ayuda para resolverlas, la cual puede ser entregada por la/el enfermera/o, que se encuentra en una posición privilegiada para apoyarlos y orientarlos, contribuyendo a aminorar los efectos perjudiciales de una práctica de crianza negativa y acrecentando los efectos positivos de una práctica de crianza efectiva.

Por último, acerca de los factores institucionales, observando la dependencia administrativa, se encontraron resultados diferentes en estudios realizados en Chile (3) y Brasil (6). Uno informa la existencia de mayor agresividad en colegios particulares, en el otro, en colegios municipalizados. El presente estudio confirmaría la investigación realizada en Brasil (6), pues se observa que un alto porcentaje de niños con alta agresividad provienen de colegios municipalizados, siendo estadísticamente significativo.

Así también, según el tipo de establecimiento, sea éste mixto o sólo de varones, el estudio muestra que un considerable porcentaje de los varones que asisten a colegios mixtos tienen un nivel alto de agresividad. No se encontró en estudios realizados la inclusión de esta variable, por lo que no es posible comparar estos resultados. Lo anterior puede explicarse dado que la gran parte de ellos fueron realizados en países que en su mayoría presentan colegios mixtos; en cambio, en nuestro país existen escuelas de los dos tipos. Este es un hallazgo no esperado, ya que el supuesto era que habría un mayor nivel de agresividad en colegios sólo de varones. Sería interesante profundizar en el estudio de este aspecto, con el propósito de 
enfocar las medidas preventivas en este tipo de colegio.

También se estudió el nivel de agresividad según tamaño del colegio al que asistían, observándose que más del 50\% de los adolescentes con nivel alto de agresividad provienen de colegios pequeños y alrededor del $40 \%$ de los varones con bajo nivel de agresividad y no agresivos asisten a colegios grandes, esto es estadísticamente significativo. El resultado es diferente a una investigación que señala que las escuelas grandes reportan niños con altos niveles de agresividad (20). Es importante señalar que en este estudio los colegios pequeños se ubican en los cerros de Valparaíso y los grandes en el plan; por lo que la ubicación geográfica sería una variable interesante de indagar.

\section{REFERENCIAS}

1. Olweus D. Conductas de acoso y amenaza entre escolares. 2a ed. Madrid: Ediciones Morata; 1998.

2. Fernández J, Serna E, expositores. Estudio transversal del uso del tiempo extraescolar en los escolares bogotanos. XIII Congreso Nacional e Internacional de recreación, equidad, inclusión y justicia social; 2004 mayo 27-29; Bogotá, Colombia: Fundación Colombiana de Tiempo Libre y Recreación; 2004.

3. Villalta M, Saavedra E, Muñoz M. "Pasado a llevar": la violencia en la educación media municipalizada. Estudios Pedagógicos. 2007; 33 (1): 45-62.

4. Nájera E. Convivencia escolar y jóvenes. Aportes de la mediación escolar a la transformación de la educación media. Ministerio de Educación, Chile - PIIE; 1999.

5. Ortega R, Mora-Merchán J. Agresividad y Violencia: el problema de la victimización entre escolares. Revista de Educación. 1997; 313: 7-27.
6. Abramovay M, Rua M. Violences in schools [Internet]. Brasil: UNESCO; 2002 [citado 15 diciembre 2008]. 330 p. Disponible en: http://unesdoc.unesco. org/images/0012/001287/128718e.pdf

7. Instituto Nacional de la Juventud. Departamento de Estudios y Evaluación. Gobierno de Chile. Tercera Encuesta Nacional de la Juventud. Chile; 2002.

8. García M, Madriaza P. Sentido y sinsentido de la violencia escolar: análisis cualitativo del discurso de estudiantes chilenos. Psykhe. 2005; 14 (1):165-180.

9. Smokowski P, Kopasz K. Bullying in School: an overview of types, effects, family characteristics, and intervention strategies. Children and Schools. 2005; 27 (2), 101-110.

10. Abramovay M. Violencia en las escuelas: un gran desafío. Revista Iberoamericana de Educación. 2005; 38: 53-66.

11. Ministerio del Interior y Ministerio de Educación. Gobierno de Chile. Segundo Estudio Nacional de violencia en el ámbito escolar: Informe encuesta alumnos. Chile; 2007.

12. Rigby K. Consequences of bullying in schools. Can J Psychiatry [Internet]. 2003 [citado 15 diciembre 2008] 48(9): 58390. Disponible en: http://ww1.cpa-apc. org/Publications/Archives/CJP/2003/ october/rigby.pdf

13. Flannery D, Wester K, Singer M. Impact of exposure to violence in school on child and adolescent mental health and behavior. J Community Psychol [Internet]. 2004 [citado 12 diciembre 2008] 32(5): 559-73. Disponible en: http:// onlinelibrary.wiley.com/doi/10.1002/ jcop.20019/abstract

14. Pinheiro P. Acabar con la violencia contra los niños, niñas y adolescentes [Internet]. ONU-UNICEF-OMS; 2006 [citado 23 mayo 2009]. 29 p. Disponible en: http:// www.educarchile.cl/UserFiles/P0001/ File/Resumen\%20estudio.pdf

15. Nansel T, Craig W, Overpeek M, Saluja 
G, Ruan W. Crossnational consistency in the relationship between bullying and psychosocial adjustment. Arch Pediatr Adolesc Med [Internet]. 2004 [citado 23 de mayo 2009] 158(8): 730-6. Disponible en: http://archpedi.ama-assn.org/cgi/ content/full/158/8/730

16. Brown V. El modelo del sistema conductual. En: Marriner A, Raile M. Modelos y teorías en enfermería. 5 a ed. Madrid, España: Elsevier; 2003.

17. Mooij T. Por la seguridad de la escuela. Revista de Educación. 1997; 313: 29-52.

18. Contador M. Percepción de la violencia escolar en estudiantes de enseñanza media. Psykhe. 2001; 10 (1): 69-80.

19. Galdames S, Arón A. Construcción de una escala para medir creencias legitimadoras de violencia en la población infantil. Psykhe. 2007; 16 (1): 15-25.

20. Funk W. Violencia escolar en Alemania: Estado del arte. Revista de Educación. 1997; 313: 53-78.

21. Campart M, Lindströn P. Intimidación y violencia en las escuelas suecas, una reseña sobre investigación y política preventiva. Revista de Educación. 1997; 313: 95-119.

22. Ramírez S, Justicia F. El maltrato entre escolares y otras conductas-problema para la convivencia. Revista Electrónica de Investigación Psicoeducativa. 2006; 9(4):265-90.

23. Brown S, Birch D, Kancherla V. Bullying perspectives: Experiences, attitudes and recommendations of 9 to 13 year olds attending health education centers in the United States. J Sch Health. 2005; 75 (10): 384-92.

24. Fierro-Hernández C. Patrón de rasgos personales y comportamiento escolar en jóvenes. Revista de Educación. 2000; 332: 291-304.

25. Echeburúa E. Personalidades violentas. Madrid, España: Ediciones Pirámide; 2003.

26. Chamberlain L, Wang Y, Robinson T.
Does Children's Screen Time Predict Requests for Advertised Products? Arch Pediatr Adolesc Med [Internet]. 2006 [citado 15 junio 2009] 160(4): 363-68. Disponible en: http://archpedi.ama-assn. org/cgi/content/full/160/4/363.

27. Ministerio del Interior. Gobierno de Chile. Consejo Nacional para el Control de Estupefacientes. Sexto Estudio Nacional de Drogas en Población Escolar de Chile $8^{\circ}$ Básico a 4 Medio, 2005; 2006.

28. Fischer K, Haas M, Watson M, Carr C. Reserved Children More Likely to Be Violent than Their Outgoing Peers. [Internet]. Cambridge, MA: Harvard Graduate School of Education [citado 15 junio 2009]. Diponible en: http:// www.gse.harvard.edu/news/features/ fischer08212002.html.

29. Ayala H, Pedroza F, Morales S, Chaparro A, Barragán N. Factores de riesgo, factores protectores y generalización del comportamiento agresivo en una muestra de niños en edad escolar. Salud mental. 2002; 25 (3): 27-39.

30. EducarChile. Violencia escolar: Un tema pendiente [Internet] Santiago, Chile: EducarChile; 2005. [citado 15 junio 2009]. Disponible en: http://www.educarchile. $\mathrm{cl} /$ Portal.Base/Web/VerContenido. aspx?ID=77826.

31. Díaz-Aguado M. Por qué se produce la violencia escolar y cómo prevenirla. Revista Iberoamericana de Educación. 2005; 37: 17-47.

32. Trautmann A. Maltrato entre pares o "bullyng". Una visión actual. Rev Chil Pediatr. 2008; 79 (1): 13-20.

33. PROTEGE: Red de Protección Social, Gobierno de Chile. Taller de habilidades para padres, madres y cuidadoras(es) de niños y niñas de 0 a 5 años [Internet]. Chile: Chile crece contigo; 2009 [citado 16 diciembre 2009]. 32 p. Disponible en: http://www.crececontigo.cl/adultos/ nadie-es-perfecto/padres-madrescuidadores/ 